\title{
A GENERAL CLASS OF FACTORS OF $E^{4}$
}

\author{
BY \\ LEONARD R. RUBIN
}

\begin{abstract}
In this paper we prove that any upper semicontinuous decomposition of $E^{n}$ which is generated by a trivial defining sequence of cubes with handles determines a factor of $E^{n+1}$. An important corollary to this result is that every 0 -dimensional point-like decomposition of $E^{3}$ determines a factor of $E^{4}$. In our approach we have simplified the construction of the sequence of shrinking homeomorphisms by eliminating the necessity of shrinking sets piecewise in a collection of $n$-cells, the technique employed by R. H. Bing in the original result of this type.
\end{abstract}

1. Introduction. In [5] Bing proved that the product of a certain nonmanifold with a line is $E^{4}$, and in [14] we proved the same was true of another space whose construction was similar in many ways to that of the "dogbone" space of [5]. Such nonmanifolds are the decomposition (quotient) spaces of upper semicontinuous decompositions of $E^{3}$ generated by trivial defining sequences whose elements are locally finite, disjoint sets of cubes with handles.

One may then ask, under what conditions do these defining sequences determine a decomposition space which is a factor of $E^{4}$ ? In [3] the authors partially answered this, generalizing the result of [14] by showing that if the defining sequence is trivial and toroidal then it determines a factor of $E^{4}$. We there conjectured that any trivial defining sequence whose elements are sets of cubes with handles defines a factor of $E^{4}$. In [15] and [16] we gave partial solutions to this conjecture; but now in this paper we shall generalize all the results of [3], [5], [14], [15], [16] by proving that the conjecture of [3] is true.

For another reference on this subject see [2]. Consult [17] for the subject of covering spaces and [9] for other references in general topology.

2. Definitions and notation. We shall use bd $(Y)$ to mean the topological boundary of a subspace $Y$ and also to mean the boundary of $Y$ as a manifold if $Y$ is a manifold. In all cases, we shall make it clear in which sense we are using the term. Similarly, for interior we shall use int $(Y)$. If $A$ is a collection of sets we shall often write $A^{*}=\bigcup\{a \mid a \in A\}$. The symbol "" will mean "homeomorphic to". Let $Z^{+}$denote the set of natural numbers, and $E^{n}$ euclidean $n$-space. We use $I$ to denote the closed unit interval $[0,1] \subset E$.

Received by the editors April 6, 1970 and, in revised form, October 14, 1970 and May 14, 1971.

AMS 1969 subject classifications. Primary 5560, 5701.

Key words and phrases. Cubes with handles, defining sequence, trivial defining sequence, universal covering space, 0-dimensional decomposition, point-like decomposition, cell-like spaces, property $\mathrm{UV}^{\infty}$. 
Let $\left\{A_{i}\right\}$ be a sequence of locally finite, disjoint collections of nonempty, compact subsets of $E^{k}$ such that, for each $i \in Z^{+}, A_{i+1}^{*} \subset \operatorname{int}\left(A_{i}^{*}\right)$. Then the collection of components of $\bigcap A_{i}^{*}$ along with the sets of points not in $\bigcap A_{i}^{*}$ is an upper semicontinuous decomposition $C$ of $E^{k}$. We say $\left\{A_{\imath}\right\}$ is a defining sequence for $C$. If for each $T \in A_{i}$, the inclusion map $\left(A_{i+1}^{*} \cap T\right) \subset T$ is null homotopic we say the defining sequence is trivial.

3. Statement of main result. This paper is devoted mainly to the proof of the following theorem.

THEOREM 1. Let $\left\{A_{i}\right\}$ be a trivial defining sequence for an upper semicontinuous decomposition $C$ of $E^{3}$. If each $A_{i}$ is a disjoint, locally finite collection of cubes with handles, then $E^{3} / C$ is a factor of $E^{4}$; specifically $\left(E^{3} / C\right) \times E \cong E^{4}$.

Before going into the details of proof it will be worthwhile to outline the approach that will be taken.

Let $S^{n}$ denote the $n$-sphere. We shall assume $S^{3}=E^{3} \cup\{\omega\}$, a one-point compactification, and that $S^{4}$ is the suspension of $S^{3}$ from the two points $N_{1}$ and $N_{2}$. Let $\Omega$ denote the arc obtained from the suspension of $\{\omega\}$ between $N_{1}$ and $N_{2}$. The suspension will be taken as the two-point compactification of $S^{3} \times E$ so that $E^{4}=S^{4}-\Omega$ and we can use the usual coordinate system of $E^{4}$.

We shall use the following notions in the sequel. Let $C$ be an upper semicontinuous decomposition of $E^{3}$ into compact elements. Then $C$ induces an upper semicontinuous decomposition of $S^{3}$ by appending $\{\omega\}$ to $C$, but we shall still refer to this decomposition as $C$. Furthermore, $C$ induces an upper semicontinuous decomposition $C^{\prime}$ of $E^{4}=E^{3} \times E$ whose elements are the sets $g \times\{t\}, g \in C$ and $t \in E$. In turn $C^{\prime}$ induces an upper semicontinuous decomposition $C^{\prime} \cup\{\{x\} \mid x \in \Omega\}$ of $S^{4}$ which we shall still call $C^{\prime}$. Suppose there exists a continuous surjective function $f: S^{4} \rightarrow S^{4}$ whose point inverses are the elements of $C^{\prime}$; then $S^{4} / C^{\prime} \cong S^{4}$. If in addition $f$ is the identity on $\Omega$ then $E^{4} / C^{\prime} \cong E^{4}$. In this case it follows from standard theory of decomposition spaces that $\left(E^{3} / C\right) \times E \cong E^{4}$ and that the suspension of $S^{3} / C$ is homeomorphic to $S^{4}$. In this paper we shall prove the existence of such a function $f$ relative to the decompositions indicated in Theorem 1. As usual, $f$ will be defined as the limit of a uniformly convergent sequence $\left\{f_{i}\right\}$ of homeomorphisms of $S^{4}$ onto itself. Each $f_{i}$ will be the identity on $\Omega$.

The standard practice is to define the sequence $\left\{f_{i}\right\}$ so that the elements of $C^{\prime}$ are uniformly shrunk to points as $i$ increases without bound. We shall prove the following lemma.

LEMMA 1. For all $\varepsilon>0$ and $i \in Z^{+}$there exists $\tilde{H}: S^{4} \cong S^{4}$ such that

(1) $\tilde{H}=1$ on the complement of $A_{i}^{*} \times E$ and in particular on $\Omega$,

(2) for all $g \in A_{i+1}$ and $t \in E$, diam $[\tilde{H}(g \times\{t\})]<\varepsilon$, and

(3) if $(x, t) \in E^{3} \times E$ and $\tilde{H}(x, t)=\left(x^{\prime}, t^{\prime}\right)$, then $\left|t^{\prime}-t\right| \leqq \varepsilon$. 
With the aid of Lemma 1 and the techniques developed in [5] and used elsewhere, the desired sequence $\left\{f_{i}\right\}$ can be constructed. We shall not give that construction here.

Lemma 1 will be a corollary of Lemma 2. For $A \subset E^{3}$ let $Z(A)$ be the first positive integer such that $Z(A)$ is greater than the distance from $A$ to the origin.

LeMma 2. For all $\varepsilon>0, i \in Z^{+}$, and $A \in A_{i}$, there exists $H: S^{4} \cong S^{4}$ such that

(1) $H=1$ on the complement of $A \times E$ and in particular on $\Omega$,

(2) for all $g \in A_{i+1}$ such that $g \subset A$, and $t \in E$, diam $[H(g \times\{t\})]<\varepsilon$, and

(3) if $(x, t) \in E^{3} \times E$ and $H(x, t)=\left(x^{\prime}, t^{\prime}\right)$, then $\left|t^{\prime}-t\right|<\varepsilon / Z(A)$.

That Lemma 1 is a corollary of Lemma 2 can be seen as follows. The map $\tilde{H}$ is to be defined piecewise by defining $\tilde{H}$ as in Lemma 2 on each set $A \times E$ for $A \in A_{i}$. Then the local finiteness of $A_{i}$ enables us to extend $\tilde{H}$ to $\left(E^{3} \times E^{1}\right) \cup\left\{N_{1}\right\} \cup\left\{N_{2}\right\}$ by setting $\tilde{H}=1$ on the complement of $A_{i}^{*} \times E$. Condition (3) guarantees that $\tilde{H}$ can be extended to $\Omega$ and is the identity on $\Omega$.

Let us describe how we plan to prove Lemma 2. Let $D$ be a 3-cell, $i \in Z^{+}$, $A \in A_{i}$ and $A_{0}=A_{i+1}^{*} \cap A$. We shall thread the tube $D \times E$ through the set $A \times E$ so that $A_{0} \times E$ is contained in its interior. We shall shrink the necessary subsets of $A_{0} \times E$ inside this tube, holding the tube fixed on its boundary and compressing in towards the central core of the tube.

To be more precise, let $\varepsilon>0$. We shall exhibit an imbedding $F: D \times E \rightarrow A \times E$ such that $\mathrm{Cl}[F(D \times E)]=F(D \times E) \cup\left\{N_{1}\right\} \cup\left\{N_{2}\right\}$ (the unique two-point compactification-thus $\mathrm{Cl}[F(D \times E)]$ is a 4-cell) having the property that $F(D \times\{t\})$ $\subset A \times[t, t+\varepsilon]$ for all $t \in E$.

The reader can easily provide a proof of the following lemma.

Lemma 3. Suppose $B$ is a compact topological space and $G: B \times E \rightarrow A \times E$ is an imbedding having the property that $G(B \times\{t\}) \subset F($ int $(D) \times\{t\})$ for all $t \in E$. Then there exists $H_{0}: S^{4} \cong S^{4}$ such that

(1) $H_{0}=1$ on the complement of $A \times E$ and in particular on $\Omega$,

(2) for all $t \in E$, diam $\left[H_{0} \circ G(B \times\{t\})\right]<\varepsilon$, and

(3) if $(x, t) \in E^{3} \times E$ and $H_{0}(x, t)=\left(x^{\prime}, t^{\prime}\right)$, then $\left|t^{\prime}-t\right|<\varepsilon$.

We shall prove the following lemma.

LEMMA 4. There exists $G: S^{4} \cong S^{4}$ such that

(1) $G=1$ on the complement of $A \times E$ and in particular on $\Omega$,

(2) $G\left(A_{0} \times\{t\}\right) \subset F$ (int $\left.(D) \times\{t\}\right)$ for all $t \in E$, and

(3) if $(x, t) \in E^{3} \times E$ and $G(x, t)=\left(x^{\prime}, t^{\prime}\right)$, then $\left|t^{\prime}-t\right|<\varepsilon$.

With appropriate choices of $\varepsilon$ in Lemmas 3 and 4, the map $H$ of Lemma 2 is the composition $H_{0} \circ G$. Therefore to prove Theorem 1, it is sufficient to demonstrate the existence of an imbedding $F: D \times E \rightarrow A \times E$ and a homeomorphism $G: S^{4} \cong S^{4}$ satisfying Lemma 4. 
4. Injecting a universal covering space. Suppose $n \geqq 1$ is a natural number and $T$ is a cube with $n$-handles ( $n$-holed solid torus). Let $F_{0}$ be a 3-cell in $T$ such that $\mathrm{Cl}\left(T-F_{0}\right)$ is the disjoint union of $n$ 3-cells, $F_{1}, \ldots, F_{n}$, where each $F_{i} \cap F_{0}$ is a disjoint pair of 2-cells. Let $p: \tilde{T} \rightarrow T$ be a universal covering projection [17] in the category of connected topological spaces.

Our purpose in this section is to show the existence of a continuous injective map (not an imbedding) $f: \tilde{T} \rightarrow T \times E$ such that $\pi \circ f=p$ where $\pi: T \times E \rightarrow T$ is the natural projection. Although the description of this map $f$ is complicated, the idea itself is not, and we have depicted schematically some of the construction in Figure 1 for a 2-holed solid torus.

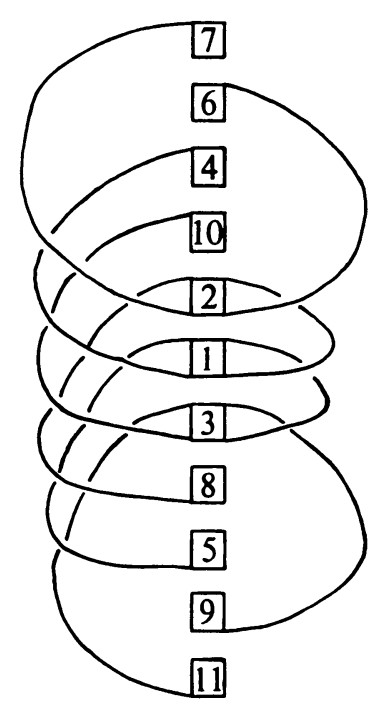

Figure 1

Each $F_{i}$ is evenly covered by $p$ in the sense that each component of $p^{-1}\left(F_{i}\right)$ maps homeomorphically onto $F_{i}$ under $p$ and is an open subset of $p^{-1}\left(F_{i}\right)$. Let $C_{i}$ denote the (countable) collection of components of $p^{-1}\left(F_{i}\right)$. Let $B$ be a 2-cell and for each $i \in\{1, \ldots, n\}$ let $g_{i}: B \times I \rightarrow F_{i}$ be a homeomorphism such that $g_{i}(B \times 0)$ and $g_{i}(B \times 1)$ are the two 2-cell components of $F_{0} \cap F_{i}$.

Write the universal covering space $\tilde{T}$ as $\bigcup\left\{\tilde{T}_{j} \mid j \in Z^{+}\right\}$where, for each $j$, $\tilde{T}_{j}$ is an element of some $C_{i}$ and $\bigcup\left\{\tilde{T}_{k} \mid 1 \leqq k \leqq j\right\}$ is a 3-cell which intersects $\tilde{T}_{j+1}$ in a 2-cell contained in one and only one $\tilde{T}_{k}$. Assume without loss of generality that $\tilde{T}_{1} \in C_{0}$, and for each $j$ let $T_{j}$ denote $p\left(\tilde{T}_{j}\right)$. If $i \neq 0$ and $T_{j}=F_{i}$, we refer to the two 2-cell components of $\left[p^{-1}\left(F_{i} \cap F_{0}\right)\right] \cap \tilde{T}_{\text {, }}$ as ends.

We will have need to use the following information.

Lemma 5. Suppose $\alpha_{1}<\beta_{1}<\alpha_{2}<\beta_{2}$ and $f_{1}, f_{2}$ are two maps of $F_{i}$ into $T \times E$ where $i \neq 0$, satisfying $f_{1}(x)=\left(x, a_{1}+\left(a_{2}-a_{1}\right) t\right)$ and $f_{2}(x)=\left(x, \beta_{1}+\left(\beta_{2}-\beta_{1}\right) t\right)$ where $x=g_{i}(b, t)$. Then both $f_{1}$ and $f_{2}$ are injective maps and $f_{1}\left(F_{i}\right) \cap f_{2}\left(F_{i}\right)=\varnothing$. 
This can be easily proved by the reader.

In what follows the reader may reference Figure 1 and find it useful to sketch the analogous construction where $T$ is replaced by a 2-cell with $n$-holes lying in the plane and $\tilde{T}$ is to be immersed in $T \times E \subset E^{3}$.

Since $\tilde{T}$ has the weak topology [9] determined by $\left\{\tilde{T}_{j}\right\}$ it will be sufficient to define $f$ piecewise on $\left\{\tilde{T}_{j}\right\}$ and we proceed by induction.

Define $f_{1}: \tilde{T}_{1} \rightarrow T \times E$ by $f_{1}(x)=(p(x), 0)$ for $x \in \tilde{T}_{1}$. Thus the condition $\pi \circ f_{1}=p$ holds on $\tilde{T}_{1}$ and $f_{1}$ is injective.

Now $\tilde{T}_{1} \cap \tilde{T}_{2}$ is a 2-cell. Let $T_{2}=F_{i}$; then $F_{i} \neq F_{0}$ by standard properties of covering spaces. Either $p\left(\tilde{T}_{1} \cap \tilde{T}_{2}\right)=g_{i}(B \times 0)$ or $g_{i}(B \times 1)$. In the former case let $\alpha_{1}=0, \alpha_{2}=1$; in the latter let $\alpha_{1}=-1, \alpha_{2}=0$. For $x=g_{i}(b, t) \in F_{i}$ define $f_{2}^{*}(x)$ $=\left(x, \alpha_{1}+\left(\alpha_{2}-\alpha_{1}\right) t\right) \in T \times E$, and for $z \in \tilde{T}_{2}$ define $f_{2}(z)=f_{2}^{*} \circ p(z)$. It is easy to check that $f_{2}$ agrees with $f_{1}$ on their common domain, the end $\tilde{T}_{1} \cap \tilde{T}_{2}$, and that $\left\{f_{1}, f_{2}\right\}$ determines an injective map of $\tilde{T}_{1} \cup \tilde{T}_{2} \rightarrow T \times E$. Furthermore, $\pi \circ f_{2}(z)$ $=p(z)$ as required.

Suppose $f_{k}$ has been defined on all $\tilde{T}_{k}$ for $k<M$ where $M>2$ and assume

(1) $\left\{f_{k} \mid k<M\right\}$ determines a well-defined, continuous, injective map of $\bigcup\left\{\tilde{T}_{k} \mid k<M\right\}$ into $T \times E$ such that $\pi \circ f_{k}(x)=p(x)$ whenever $x \in \tilde{T}_{k}$,

(2) if $T_{k}=F_{0}$ then $f_{k}\left(\tilde{T}_{k}\right) \subset T \times \alpha$ for some $\alpha \in E$,

(3) if $K \subset \tilde{T}_{k}$ is an end then $f_{k}(K) \subset T \times \alpha$ for some $\alpha \in E$,

(4) if $T_{k}=F_{i}, i \neq 0$, then there are numbers $\alpha_{1}<\alpha_{2}$ and a map $f_{k}^{*}: F_{i} \rightarrow T \times E$ given by $f_{k}^{*}(x)=\left(x, \alpha_{1}+\left(\alpha_{2}-\alpha_{1}\right) t\right)$ where $x=g_{i}(b, t)$ and $f_{k}=f_{k}^{*} \circ p$ with $p$ restricted to $\tilde{T}_{k}$.

Let $T_{M}=F_{i}$. There is one and only one $j<M$ for which $\tilde{T}_{M} \cap \tilde{T}_{j} \neq \varnothing$. Then $\tilde{T}_{M} \cap \tilde{T}_{j}$ is an end. Let $\alpha_{1}$ be the real number for which $f_{j}\left(\tilde{T}_{M} \cap \tilde{T}_{j}\right) \subset T \times \alpha_{1}$. We break the construction of $f_{M}$ into two cases.

Case 1. $F_{i}=F_{0}$. Then define $f_{M}(x)=\left(p(x), \alpha_{1}\right)$ for all $x \in \tilde{T}_{M}$. It is easy to verify that with this definition of $f_{M},\left\{f_{k} \mid k<M+1\right\}$ satisfies all the conditions of the induction hypothesis.

Case 2. $F_{i} \neq F_{0}$. Let $W=\left\{s \mid\right.$ for some $j<M$ and some end $K$ of $\left.\tilde{T}_{j}, f_{j}(K) \subset T \times s\right\}$. Then let $V=\left\{\left(\beta, \beta^{\prime}\right) \mid \beta<\alpha_{1}<\beta^{\prime}\right.$ and for some $j<M$ there is a $\tilde{T}_{j} \in C_{i}$ having ends $K, K^{\prime}$ with $f_{j}(K) \subset T \times \beta$ and $\left.f_{j}\left(K^{\prime}\right) \subset T \times \beta^{\prime}\right\}$. The following may easily be checked. If $k<M, \tilde{T}_{k} \in C_{i}$, and the ends of $\tilde{T}_{k}$ map to $T \times \sigma_{1}, T \times \sigma_{2}$ where $\sigma_{1}<\sigma_{2}$, then it is impossible that $\left(\beta, \beta^{\prime}\right) \in V$ and $\beta<\sigma_{1}<\sigma_{2} \leqq \beta^{\prime}$. If this were true, it would not be difficult to show the maps $\left\{f_{k} \mid k<M\right\}$ did not determine an injective map on $\bigcup\left\{T_{k} \mid k<M\right\}$.

There are now two possibilities, either $p\left(\tilde{T}_{M} \cap \tilde{T}_{j}\right)=g_{i}(B \times 0)$ or $g_{i}(B \times 1)$. We shall consider $p\left(\tilde{T}_{M} \cap \tilde{T}_{j}\right)=g_{i}(B \times 0)$ only, the latter situation requiring similar but symmetric techniques.

If $V=\varnothing$ let $\sigma=\inf \left[\left\{s \mid s \in W\right.\right.$ and $\left.\left.s>\alpha_{1}\right\} \cup\left\{\alpha_{1}+1\right\}\right]$. Let $\alpha_{2}$ be a real number such that $\alpha_{1}<\alpha_{2}<\sigma$. With this choice, if $k<M$ and $T_{k}=K_{i}$, then $f_{k}\left(\tilde{T}_{k}\right)$ $\cap\left(T \times\left[\alpha_{1}, \alpha_{2}\right]\right)=\varnothing$. We shall define $f_{M}: \tilde{T}_{M} \rightarrow T \times\left[\alpha_{1}, \alpha_{2}\right]$ so that it agrees with $f_{j}$ 
on $\tilde{T}_{M} \cap \tilde{T}_{j}$. Define $f_{M}^{*}: F_{i} \rightarrow T \times\left[\alpha_{1}, \alpha_{2}\right]$ by the rule $f_{M}^{*}(x)=\left(x, \alpha_{1}+\left(\alpha_{2}-\alpha_{1}\right) t\right)$ where $x=g_{i}(b, t)$. Then define $f_{M}(z)=f_{M}^{*} \circ p(z)$ for $z \in \tilde{T}_{M}$.

However, if $V \neq \varnothing$ we must choose $\alpha_{2}$ more carefully. Let $\left(\beta_{1}, \beta_{2}\right) \in V$ such that if $\left(\beta, \beta^{\prime}\right) \in V$ then $\beta \leqq \beta_{1}$. Let $\sigma=\inf \left[\left\{s \mid s \in W\right.\right.$ and $\left.\left.s>\beta_{2}\right\} \cup\left\{\beta_{2}+1\right\}\right]$. Choose $\alpha_{2}$ so that $\beta_{2}<\alpha_{2}<\sigma$. With this choice of $\alpha_{2}$, define $f_{M}$ as for the case $V \neq \varnothing$.

There is no difficulty seeing $f_{M}$ is injective and that $f_{M}$ agrees with $f_{j}$ on $\tilde{T}_{M} \cap \tilde{T}_{j}$. Therefore $\left\{f_{k} \mid k \leqq M\right\}$ uniquely determines a map of $\bigcup\left\{\tilde{T}_{k} \mid k \leqq M\right\}$ to $T \times E$. We now indicate why this map is injective.

By our choice of $\alpha_{2}$, and since $f_{M}\left(\widetilde{T}_{M}\right) \subset F_{i} \times E$, the only way $\left\{f_{k} \mid k \leqq M\right\}$ may not determine an injective map is that for some $q<M, \tilde{T}_{q} \in C_{i}$ and $f_{q}\left(\tilde{T}_{q}\right) \cap f_{M}\left(\tilde{T}_{M}\right) \neq \varnothing$. Suppose $\tilde{T}_{q}$ has ends $K, K^{\prime}$ for which $f_{q}(K) \subset T \times \beta$ and $f_{q}\left(K^{\prime}\right) \subset T \times \beta^{\prime}$. If $\left(\beta, \beta^{\prime}\right) \in V$, it is not true that $\beta<\beta_{1} \leqq \beta_{2} \leqq \beta^{\prime}$; since $\beta \leqq \beta_{1}$, then $\beta<\beta_{1}<\beta^{\prime}<\beta_{2}$. Hence, $\beta<\alpha_{1}<\beta^{\prime}$ $<\alpha_{2}$, so that, by Lemma $5, f_{q}^{*}\left(T_{q}\right) \cap f_{M}^{*}\left(T_{M}\right)=\varnothing$. Therefore $f_{q}\left(\tilde{T}_{q}\right) \cap f_{M}\left(\tilde{T}_{M}\right)=\varnothing$.

If $\left(\beta, \beta^{\prime}\right) \notin V$ then either

(1) $\beta<\beta^{\prime}<\alpha_{1}<\alpha_{2}$,

(2) $\alpha_{1}<\beta<\beta^{\prime}<\alpha_{2}$, or

(3) $\alpha_{1}<\alpha_{2}<\beta<\beta^{\prime}$.

A simple analysis will rule out (2). In (1) and (3) since $f_{q}^{*}\left(T_{q}\right) \subset T \times\left[\beta, \beta^{\prime}\right]$ and $f_{M}^{*}\left(T_{M}\right) \subset T \times\left[\alpha_{1}, \alpha_{2}\right], f_{q}\left(\tilde{T}_{q}\right) \cap f_{M}\left(\tilde{T}_{M}\right)=\varnothing$.

5. Some 4-cells in $T \times E$. Let $M \in Z^{+}, D=\bigcup\left\{\tilde{T}_{k} \mid k \leqq M\right\}$, and $f$ also denote the restriction of $f$ to $D$. Because $f$ is an injection and $D$ is compact, the next lemma is true.

LEMMA 6. The map $f: D \rightarrow T \times E$ is an imbedding.

For each $\theta \in E$, let $L_{\theta}: T \times E \cong T \times E$ be the map which sends $(x, t)$ to $(x, t+\theta)$. Then define $f_{\theta}: D \rightarrow T \times E$ by $f_{\theta}=L_{\theta} \circ f$. Define $F^{*}: D \times E \rightarrow T \times E$ by $F^{*}(x, \theta)$ $=f_{\theta}(x)$ and $F^{* *}: D \times E \rightarrow T \times E \times E$ by $F^{* *}(x, \theta)=\left(F^{*}(x, \theta), \theta\right)$.

LEMmA 7. There exists $\varepsilon>0$ such that if $\alpha<\theta$ and $\theta-\alpha \leqq \varepsilon$, then $F^{*}$ on $D \times[\alpha, \theta]$ is an imbedding.

Proof. First note that if $k \leqq M$ then $F^{*}$ restricted to $\tilde{T}_{k} \times E$ is injective. This may be easily computed by the reader from the definition of $F^{*}$ since $f$ on $\tilde{T}_{k}$ is injective.

Suppose $k \neq j$ and $\left\{\tilde{T}_{k}, \tilde{T}_{j}\right\} \subset C_{i}$ for some $i$. Then $T_{k}=T_{j}=F_{i}, f\left(\tilde{T}_{k}\right)=f_{k}^{*}\left(F_{i}\right)$, and $f\left(\tilde{T}_{j}\right)=f_{j}^{*}\left(F_{i}\right)$. Associated with $f_{k}^{*}$ are numbers $\alpha_{1}<\alpha_{2}$ and with $f_{j}^{*}$ are numbers $\beta_{1}<\beta_{2}$ where either $\left[\alpha_{1}, \alpha_{2}\right] \cap\left[\beta_{1}, \beta_{2}\right]=\varnothing$, or $\alpha_{1}<\beta_{1}<\alpha_{2}<\beta_{2}$, or $\beta_{1}<\alpha_{1}<\beta_{2}<\alpha_{2}$. In any case, if we examine the definitions of the maps $f_{k}^{*}, f_{j}^{*}$ we can see there exists a number $\varepsilon>0$ such that if $\sigma \leqq \varepsilon$ and $x=g_{i}(b, t) \in F_{i}$, then $L_{\sigma} \circ f_{k}^{*}(x)$ $=\left(x, \alpha_{1}+\left(\alpha_{2}-\alpha_{1}\right) t+\sigma\right) \neq\left(x, \beta_{1}+\left(\beta_{2}-\beta_{1}\right) t+\sigma\right)=L_{\sigma} \circ f_{j}^{*}(x)$. For example, in case $\beta_{1}<\alpha_{1}<\beta_{2}<\alpha_{2}$, a formal computation shows that $\varepsilon=\min \left\{\left(\alpha_{1}-\beta_{1}\right) / 2,\left(\alpha_{2}-\beta_{2}\right) / 2\right\}$ will suffice.

Thus $L_{\sigma} \circ f_{k}^{*}\left(F_{i}\right) \cap L_{\sigma} \circ f_{j}^{*}\left(F_{i}\right)=\varnothing$. Therefore $L_{\sigma} \circ f\left(\tilde{T}_{k}\right) \cap L_{\sigma} \circ f\left(\tilde{T}_{j}\right)=\varnothing$, so 
$f_{\sigma}\left(\tilde{T}_{k}\right) \cap f_{\sigma}\left(\tilde{T}_{j}\right)=\varnothing$. We conclude further that $F^{*}\left(\tilde{T}_{k} \times[\alpha, \theta]\right) \cap F^{*}\left(\tilde{T}_{j} \times[\alpha, \theta]\right)=\varnothing$ as long as $\theta-\alpha<\varepsilon$.

By induction on the finite number of $\tilde{T}_{k} \in C_{i}$ we see there exists $\varepsilon>0$ such that, for any pair $\left\{\tilde{T}_{k}, \tilde{T}_{j}\right\} \subset C_{i}, F^{*}\left(\tilde{T}_{k} \times[\alpha, \theta]\right) \cap F^{*}\left(\tilde{T}_{j} \times[\alpha, \theta]\right)=\varnothing$, as long as $\theta-\alpha<\varepsilon$. Let $S_{i}=\bigcup\left\{\tilde{T}_{k} \mid k \leqq M\right.$ and $\left.\tilde{T}_{k} \in C_{i}\right\}$. Then $F^{*}$ restricted to $S_{i} \times[\alpha, \theta]$ is injective as long as $\theta-\alpha<\varepsilon$. Since we may do the above for each $S_{i}$, we may choose $\varepsilon$ to be the minimum of the finite set of numbers $\varepsilon$ so obtained, one for each $S_{i}$. Now if $\theta-\alpha<\varepsilon, \bigcup F^{*}\left(S_{i} \times[\alpha, \theta]\right)=F^{*}(D \times[\alpha, \theta])$ so that $F^{*}$ is injective on the compact set $D \times[\alpha, \theta]$ and is therefore an imbedding of it.

Roughly speaking, $F^{*}$ on $D \times[\alpha, \theta]$ is an imbedding obtained by stacking copies of $f(D)$.

One may similarly deduce the following lemma which is important to us in the sequel.

LEMMA 8. There exists a number $\varepsilon>0$ satisfying the following conditions. Suppose $x, y \in D, x \in \tilde{T}_{k}, y \in \tilde{T}_{l}, \tilde{T}_{k} \cap \tilde{T}_{l}=\varnothing$. Let $f(x)=(\bar{x}, u)$ and $f(y)=(\bar{y}, v)$ and suppose $\bar{x}, \bar{y} \in F_{i}$. If $i=0$, then $|u-v|>\varepsilon$. If $i \neq 0$, but both $\bar{x}, \bar{y} \in g_{i}(B \times a)$ for some $a \in I$, then $|u-v|>\varepsilon$.

6. A pseudo-isotopy in a cube with handles. Let $A$ be a cube with $n$-handles and $X$ a compact subset of int $(A)$. Let $T \subset$ int $(A)$ be a cube with $n$-handles obtained from $A$ by moving away from the boundary of $A$ along a collar so that $X \subset \operatorname{int}(T)$. To be more precise, there is an imbedding of bd $(A) \times I$ into $A$ such that $(x, 0) \rightarrow x$ and, for any $t>0,(x, t)$ is mapped into int $(A)$. Then for some $\varepsilon>0, T$ may be taken to be the closure of the complementary domain of the image of bd $(A) \times \varepsilon$ which does not contain bd $(A)$.

Let $T$ be written as the union of 3-cells, $F_{0}, F_{1}, \ldots, F_{n}$, as in $\S 4$.

We wish to state the existence of a certain map $\mu: A \times[-1,1] \rightarrow A$, and for this purpose it is best to think of $A$ as a standard, unknotted, unlinked cube with $n$-handles lying in $E^{3}$. Thus, if $K$ is a cell with $n$-holes lying in $E^{2}$, we might use $A=K \times I \subset E^{2} \times E=E^{3}$. Then, by moving away from bd $(K)$ along a collar as we did with $A$, we find a cell with $n$ holes, say $W \subset$ int $(K)$. Take $T=W \times\left[\frac{1}{2}, \frac{3}{4}\right]$. The following lemma is obvious.

Lemma 9. There exists a continuous map $\mu: A \times[-1,1] \rightarrow A$ satisfying the following conditions:

(1) for $t \in[-1,1]$, the map $\mu_{t}=\left.\mu\right|_{A \times t}$ is the identity map on bd $(A)$,

(2) for $t \in(-1,1), \mu_{t}$ is a homeomorphism,

(3) $\mu_{-1}(T) \cap \mu_{t}(T)=\varnothing$ for $t \neq-1$ and $\mu_{1}(T) \cap \mu_{t}(T)=\varnothing$ for $t \neq 1$,

(4) if $\bar{x}, \bar{y} \in T$ and $\mu(\bar{x}, t)=\mu(\bar{y}, u)$ then for some $i$, both $\bar{x}, \bar{y} \in F_{i}$, and furthermore, if $i \neq 0$, then there is a number $a \in I$ such that both $\bar{x}, \bar{y} \in g_{i}(B \times a)$,

(5) if $a, b \in[-1,1], a \neq b$, then $\mu_{a}(\bar{x}) \neq \mu_{b}(\bar{x})$ for all $\bar{x} \in T$,

(6) both $\mu_{1}(T)$ and $\mu_{-1}(T)$ are topologically equivalent to $K$ above, a cell with $n$ holes. 
Intuitively we may think that as $t \rightarrow 1$, the maps $\mu_{t}$ move $T$ away from itself and upwards, gradually thinning $T$ until it reaches 0 thickness at $t=1$. There is a similar description for $t \rightarrow-1$. The map $\mu$ may be referred to as a pseudo-isotopy.

7. A certain imbedding. Let $A$ and $T$ be as in the previous section, $f, \tilde{T}$, etc., as in $\S 4$ and $D=\bigcup\left\{\tilde{T}_{k} \mid k \leqq M\right\}$ for some $M \in Z^{+}$. We shall now determine an imbedding $F: D \times E \rightarrow A \times E$ as promised in $\S 3$ by adjusting the map $F^{*}$ of $\S 5$.

We shall adjust $F^{*}$ relative to the map $\mu$ of Lemma 9 .

Let $\left\{\alpha_{i} \mid i\right.$ an integer $\} \subset(-1,1)$ be a sequence such that if $i<j$, then $\alpha_{i}<\alpha_{j}$, $\mu_{x}(T) \cap \mu_{y}(T)=\varnothing$ whenever $x \leqq \alpha_{i}$ and $\alpha_{j} \leqq y$, inf $\left\{\alpha_{i}\right\}=-1$ and $\sup \left\{\alpha_{i}\right\}=1$. Let $\varepsilon>0$ be as in Lemma $8, \delta=\varepsilon / 2$, and $\eta: E \cong(-1,1)$ such that, for each integer $n$, $\eta(n \delta)=\alpha_{n}$ and $\eta$ carries the closed interval $[n \delta,(n+1) \delta]$ linearly onto $\left[\alpha_{n}, \alpha_{n+1}\right]$.

Let us now define the function $F: D \times E \rightarrow A \times E$. If $(x, t) \in D \times E$, then $x \in D$, so $f(x)=(\bar{x}, u) \in T \times E \subset A \times E$. Define $F(x, t)=\left(\mu_{n t}(\bar{x}), u+t\right)$. To see $F$ is continuous, observe that $F$ is equivalent to the composition of functions indicated as follows:

$$
\begin{aligned}
D \times E \stackrel{F^{* *}}{\longrightarrow} T \times E \times E \stackrel{1 \times \eta}{\longrightarrow} A & \times E \times(-1,1) \\
& \cong A \times(-1,1) \times E \subset A \times I \times E \stackrel{\mu \times 1}{\longrightarrow} A \times E .
\end{aligned}
$$

To prove $F$ is an imbedding it is only necessary to show $F$ is injective. To this end suppose $(x, t),(y, s) \in D \times E,(x, t) \neq(y, s)$ and $F(x, t)=\left(\mu_{\eta t}(\bar{x}), u+t\right)$ $=\left(\mu_{n s}(\bar{y}), v+s\right)=F(y, s)$, where $f(x)=(\bar{x}, u)$ and $f(y)=(\bar{y}, v)$. Then $\mu_{\eta t}(\bar{x})=\mu_{n s}(\bar{y})$ and $u+t=v+s$. We shall first conclude that $\bar{x} \neq \bar{y}, t \neq s$, and $u \neq v$.

If $\bar{x}=\bar{y}$ then by Lemma 9(5) it must be true that $\eta t=\eta s$ so that $t=s$. This implies $u=v$ so that $(\bar{x}, u)=(\bar{y}, v)$. Since $f(x)=(\bar{x}, u), f(y)=(\bar{y}, v)$, and $f$ is injective, $x=y$. Therefore $(x, t)=(y, s)$ which is a contradiction, so we conclude $\bar{x} \neq \bar{y}$.

Suppose $t=s$; then $\eta t=\eta s$. Since $\mu_{\eta t}$ is injective by Lemma $9(2)$, and $\bar{x} \neq \bar{y}$, then $\mu_{\eta t}(\bar{x}) \neq \mu_{n t}(\bar{y})=\mu_{n s}(\bar{y})$, again a contradiction. So $t \neq s$ and hence $u \neq v$.

Assume $t>s$ so that either $t-s>\varepsilon$ or $0<t-s \leqq \varepsilon$. If $t-s>\varepsilon$, then $\mu_{n t}(T) \cap \mu_{n s}(T)$ $=\varnothing$ and, since both $\bar{x}, \bar{y} \in T, \mu_{n t}(\bar{x}) \neq \mu_{n s}(\bar{y})$. This leaves only the possibility that $0<t-s \leqq \varepsilon$. Since $\mu_{n t}(\bar{x})=\mu_{n s}(\bar{y})$, by Lemma $9(4)$ both $\bar{x}, \bar{y} \in F_{i}$ for some $0 \leqq i \leqq n$. If $i=0$, then both $\bar{x}, \bar{y} \in F_{0}$. Since $u \neq v,|u-v|>\varepsilon$. But $0=u+t-v-s=(t-s)$ $+(u-v)$. Hence $t-s=v-u$, so $|t-s|=t-s=|u-v|$ which is a contradiction. It must be concluded then that $i \neq 0$. In this case, by Lemma $9(4)$ there exists a number $a \in I$ such that both $\bar{x}, \bar{y} \in g_{i}(B \times a)$. This again implies $|u-v|>\varepsilon$ which leads to a contradiction and completes the proof that $F$ is injective.

The reader may desire a better intuitive idea for the last case, $0<t-s \leqq \varepsilon$. The basic concept is that $F^{*}$ on $D \times[s, t]$ is an imbedding and that $F(D \times[s, t])$ is obtained by adjusting the 4-cell $F^{*}(D \times[s, t])$ continuously with respect to $\mu$.

Define $\mu^{*}: A \times E \cong A \times E$ by the rule $\mu^{*}(x, t)=\left(\mu_{\eta}(x), t\right)$. The map $\mu^{*}=1$ on bd $(A) \times E$. We can now state the important properties of the imbedding $F$. 
Lemma 10. Let $A$ be a cube with handles and $X$ a compact subset of int $(A)$. There exists $T \subset \operatorname{int}(A)$ with $T \cong A$ and $X \subset \operatorname{int}(T)$. Furthermore if $D=\bigcup\left\{\tilde{T}_{k} \mid k \leqq M\right\}$ is a 3-cell in $\tilde{T}$, there is an imbedding $F: D \times E \rightarrow A \times E$ such that, for each $t \in E$, $\pi \circ F(D \times t) \subset \pi \circ \mu^{*}(T \times t) \subset \operatorname{int}\left(\pi \circ \mu^{*}(A \times T)\right)=\operatorname{int}(A)$ where $\pi: A \times E \rightarrow A$ is the natural projection. If $\varepsilon>0$ we may select $F$ to also have the property that, for each $t \in E$, the projection of $F(D \times t) \subset A \times E$ into $E$ is contained in the interval $[t, t+\varepsilon]$.

8. Adjusting $E^{4}$. Let $A$ be a cube with handles and $X$ be a compact subset of int $(A)$ such that the inclusion $X \subset \operatorname{int}(A)$ is null homotopic. Then choosing $T \subset \operatorname{int}(A)$ as in $\S 6, X \subset \operatorname{int}(A)$ and the inclusion $X \subset \operatorname{int}(T)$ is null homotopic since $T$ is a strong deformation retract of $A$. We now proceed as in [3]. According to the homotopy lifting theorem [17] there is a lifting imbedding $L$ of $X$ into $\tilde{T}$. So for any $x \in X, p \circ L(x)=x$. Since $L(X)$ is compact there exists $M \in Z^{+}$for which $L(X) \subset \operatorname{int}(D)$ where $D=\bigcup\left\{\tilde{T}_{k} \mid k \leqq M\right\}$ which is a 3-cell. Then $f \circ L$ is an imbedding of $X$ into $f($ int $(D)) \subset f(D)$. There is a lifting homeomorphism $\lambda: A \times E$ $\rightarrow A \times E$ defined as in $\$ 2$ of [3] having the properties:

(1) $\lambda=1$ on the complement of $T \times E$, and

(2) if $\theta \in E$ then $\lambda(X \times \theta)=f_{\theta} \circ L(X)$ where $f_{\theta}$ is as in $\S 5$.

Furthermore if $\varepsilon>0$ we may choose $\lambda$ so that it changes $E$ coordinates no more than $\varepsilon$.

Substitute $A_{0}$ for $X$ in the hypothesis of Lemma 4. Define $G: S^{4} \cong S^{4}$ by $G=\mu^{*} \circ \lambda$ on $A \times E$ and the identity elsewhere. Then it is easy to check that $G$ satisfies all the requirements of Lemma 4 as stated in $\$ 3$. Therefore the main result of this paper, Theorem 1, is established.

9. Further results. By examining the steps in the proof of Theorem 1, and in particular the construction of the map $f$, it is not difficult to see that certain of the dimensional restrictions were not necessary. Using $k$-cells with handles in place of cubes with handles we can state a more general theorem.

THEOREM 2. Let $\left\{A_{i}\right\}$ be a trivial defining sequence for an upper semicontinuous decomposition $C$ of $E^{k}(k \geqq 3)$. If each $A_{i}$ is a disjoint, locally finite collection of $k$-cells with handles, then $\left(E^{k} / C\right) \times E \cong E^{k+1}$.

By Theorem 1 of [11] if $C$ is a point-like 0-dimensional decomposition of $E^{3}$, then $C$ is definable by cubes with handles. It is not difficult to see that because $C$ is point-like, $C$ is also definable by a trivial sequence of cubes with handles. However, recent developments allow us to state even more. Recall [10] that $A$ is cell-like if there is an imbedding $f$ of $A$ into some euclidean space such that $f(A)$ is cellular. Since $E^{n}$ is an ANR, by Theorem 1.1 of [10], a subset $A$ of $E^{n}$ is cell-like if and only if it has the property $\mathrm{UV}^{\infty}[12]$ with respect to $E^{n}$.

Now suppose $C$ is an upper semicontinuous decomposition of $E^{n}$ having the property that the closure of the projection (to the decomposition space) of the union of the nondegenerate elements of $C$ can be written as a disjoint union of compact sets $\left\{C_{\alpha}\right\}$ such that each $C_{\alpha}$ is 0 -dimensional and $\left\{C_{\alpha}\right\}$ is locally finite. 
If, in addition, each element of $C$ is cell-like, then we shall say $C$ is a standard celllike decomposition of $E^{n}$. (By the comments above, it would be equivalent to say each element of $C$ has property $U^{\infty}{ }^{\infty}$ with respect to $E^{n}$.) Referring to [12] and the proof of Theorem 1 of [11], we see that if $C$ is a standard cell-like decomposition of $E^{3}$, then $C$ is definable by a trivial sequence of cubes with handles. The following theorem and corollary follow from the preceding remarks and Theorem 2.

THEOREM 3. Let $C$ be a standard cell-like decomposition of $E^{3}$. Then $\left(E^{3} / C\right)$ $\times E \cong E^{4}$.

COROLlaRY. Every point-like 0-dimensional decomposition of $E^{3}$ determines a factor of $E^{4}$.

The following conjecture has been partially solved in [7] and [8].

CONJECTURE. Let $C$ be a standard cell-like decomposition of $E^{n}$. Then $\left(E^{n} / C\right)$ $\times E \cong E^{n+1}$.

The results of [1] may be useful in attacking this problem.

\section{REFERENCES}

1. W. R. Alford and R. B. Sher, Defining sequences for compact 0-dimensional decompositions of $E^{n}$, Bull. Acad. Polon. Sci. Sér. Sci. Math. Astronom. Phys. 17 (1969), 209-212. MR 40 \#8031.

2. J. J. Andrews and M. L. Curtis, n-space modulo an arc, Ann. of Math. (2) 75 (1962), 1-7. MR 25 \#2590.

3. J. J. Andrews and Leonard Rubin, Some spaces whose product with $E^{1}$ is $E^{4}$, Bull. Amer. Math. Soc. 71 (1965), 675-677. MR 31 \#726.

4. R. H. Bing, $A$ decomposition of $E^{3}$ into points and tame arcs such that the decomposition space is topologically different from $E^{3}$, Ann. of Math. (2) 65 (1957), 484-500. MR 19, 1187.

5. - The cartesian product of a certain nonmanifold and a line is $E^{4}$, Ann. of Math. (2) 70 (1959), 399-412. MR 21 \#5953.

6. - Point-like decompositions of $E^{3}$, Fund. Math. 50 (1961/62), 431-453. MR $25 \# 560$.

7. J. L. Bryant, Euclidean space modulo a cell, Fund. Math. 63 (1968), 43-51. MR 37 \#5861.

8. - Euclidean $n$-space modulo an $(n-1)$-cell (to appear).

9. J. Dugundji, Topology, Allyn and Bacon, Boston, Mass., 1966. MR 33 \#1824.

10. R. C. Lacher, Cell-like mappings. I, Pacific J. Math. 30 (1969), 717-731. MR 40 \#4941.

11. H. W. Lambert and R. B. Sher, Point-like 0-dimensional decompositions of $S^{3}$, Pacific J. Math. 24 (1968), 511-518. MR 37 \#902.

12. D. R. McMillan, Jr., A criterion for cellularity in a manifold, Ann. of Math. (2) 79 (1964), 327-337. MR 28 \#4528.

13. R. H. Rosen, $E^{4}$ is the Cartesian product of a totally non-euclidean space and $E^{1}$, Ann. of Math. (2) 73 (1961), 349-361. MR 23 \#A2198.

14. L. R. Rubin, The product of an unusual decomposition space with a line is $E^{4}$, Duke Math. J. 33 (1966), 323-329. MR 33 \#3283.

15. - The product of any dogbone space with a line is $E^{4}$, Duke Math. J. 37 (1970), 189-192.

16. - Recognizing certain factors of $E^{4}$, Proc. Amer. Math. Soc. 26 (1970), 199-200. MR 42 \#1088.

17. E. H. Spanier, Algebraic topology, McGraw-Hill, New York, 1966. MR 35 \#1007.

Department of Mathematics, University of Oklahoma, Norman, Oklahoma 73069 